\title{
Dispersion of aerosol particles in the free atmosphere using ensemble forecasts
}

\author{
T. Haszpra ${ }^{1}$, I. Lagzi ${ }^{2}$, and T. Tél ${ }^{1}$ \\ ${ }^{1}$ Institute of Theoretical Physics, and MTA-ELTE Research Group in Theoretical Physics, Eötvös Loránd University, \\ Pázmány P. s. 1/A, Budapest, 1117, Hungary \\ ${ }^{2}$ Department of Meteorology, Eötvös Loránd University, Budapest, and Department of Physics, \\ Budapest University of Technology and Economics, Budafoki út 8., Budapest, 1111, Hungary
}

Correspondence to: T. Haszpra (hatimi@ caesar.elte.hu)

Received: 29 May 2013 - Accepted: 2 September 2013 - Published: 14 October 2013

\begin{abstract}
The dispersion of aerosol particle pollutants is studied using 50 members of an ensemble forecast in the example of a hypothetical free atmospheric emission above Fukushima over a period of 2.5 days. Considerable differences are found among the dispersion predictions of the different ensemble members, as well as between the ensemble mean and the deterministic result at the end of the observation period. The variance is found to decrease with the particle size. The geographical area where a threshold concentration is exceeded in at least one ensemble member expands to a 5-10 times larger region than the area from the deterministic forecast, both for air column "concentration" and in the "deposition" field. We demonstrate that the rootmean-square distance of any particle from its own clones in the ensemble members can reach values on the order of one thousand kilometers. Even the centers of mass of the particle cloud of the ensemble members deviate considerably from that obtained by the deterministic forecast. All these indicate that an investigation of the dispersion of aerosol particles in the spirit of ensemble forecast contains useful hints for the improvement of risk assessment.
\end{abstract}

\section{Introduction}

In the case of anthropogenic emissions, industrial accidents or natural disasters, when different gases or aerosol particles get into the atmosphere, it is important to predict the dispersion of air pollutants for the subsequent days. There are several dispersion models that can simulate the movement of air pollutants in the atmosphere by using meteorological weather prediction data. The case of aerosol particles is of special importance (e.g., volcanic eruptions, power plant accidents). In this paper we concentrate on the effect of the variability of a forecast on the particle dispersion.

Within a weather forecast there are two main sources of uncertainties. The first one derives from the limitation of accuracy in the measurements, lack of data, erroneous data, and the sparse, unevenly distributed observation system. The approximations result in imperfect description of the meteorological initial conditions for the weather prediction model, and these errors increase in time. The second error source is the limitation of the numerical weather prediction models: imperfection in the model formulation and parameterization of some physical processes, approximate mathematical methods to solve the equation system numerically. To unfold some of these uncertainties, in ensemble forecasts (Molteni et al., 1996; Kalnay, 2003; Hágel and Horányi, 2007; Leutbecher and Palmer, 2008; Ihász et al., 2010) a weather prediction model is run with slightly perturbed, equally possible initial conditions or parameterization. If the ensemble members remain close to each other, the forecast is most likely reliable; otherwise the ensemble forecasts provide a possible range of the development of the weather. The other benefit of multiple numerical predictions is that ensemble forecasts can give the estimates of the probability of different weather events. In the calculation of air pollutant dispersion, another error source might also show up in connection with the dispersion model itself: parameterization of numerous processes (turbulent diffusion, dry and wet deposition, radioactive decay), interpolation, numerical schemes to solve the equation of motion of air pollutants. 
Galmarini et al. (2004) summarize the possible sources of uncertainties of ensemble dispersion forecasting.

Quantifying the uncertainties in the dispersion of gas pollutants (ideal tracers) due to uncertainties of the forecasts has been investigated in various ways. Dabberdt and Miller (2000) considered an ensemble of slightly different meteorological fields and the related surface concentration fields of $\mathrm{H}_{2} \mathrm{SO}_{4}$. In other cases the effects of different physical parameterization schemes of the meteorological model on dispersion were studied in Lagrangian dispersion models, like SCIPUFF and HYSPLIT (Warner et al., 2002; Challa et al., 2008). Several papers revealed the relation between standard ensemble forecasts and uncertainties in dispersion patterns. In all cases a large spread in the predicted pollutant distributions was found (Scheele and Siegmund, 2001; Straume et al., 1998; Straume, 2001; Holt et al., 2009; Lee et al., 2009).

To our knowledge no systematic investigation of the dispersion of aerosol particles has been carried out in ensemble forecasts. We therefore choose a simple dispersion model based on the simulation of Newton's equation for each particle instead of more complex schemes, like, for example, FLEXPART (Stohl et al., 2005). A further simplification is that the simulations are stopped on the top of the boundary layer at an average height of $850 \mathrm{hPa}$. In this first attempt, the uncertainties arising from turbulent diffusion and wet deposition, which would be relevant within the boundary layer only, are thus not taken into account. We emphasize that our work is only meant to be a demonstration of the variability of dispersion predictions calculated using different ensemble members of a representative meteorological forecast.

After the Chernobyl accident, most agencies developed various accidental release models coupled to weather prediction models (Galmarini et al., 2001). The performance of these models was evaluated against the ETEX European tracer experiments (Dop et al., 1998). The ETEX experiment was an international tracer campaign during which a passive tracer was tracked over a large part of Europe for several days from its release in France by monitoring at 168 groundlevel sampling stations. The main conclusion from these investigations was that one of the most crucial inputs to any dispersion model is the resolution and the quality of meteorological data and boundary layer descriptions. The importance of the horizontal resolution of meteorological data has been demonstrated by many of the simulations (Nasstrom and Pace, 1998). In a study the authors showed that the double structure of the first ETEX plume was picked up by their model when using fine-resolution weather predictions but not with meteorological data of coarser resolution (Sørensen, 1998). We therefore intend to use here ECMWF forecasts with the best resolution available.

In our study individual spherical particles with fixed, realistic radius $(r=0-10 \mu \mathrm{m})$ and density $\left(\rho_{\mathrm{p}}=2000 \mathrm{~kg} \mathrm{~m}^{-3}\right)$ are followed in a Lagrangian way. To illustrate our concept, we analyze the dispersion pattern of aerosol particles from a hypothetical puff of emission from the Fukushima Nuclear Power Plant using wind forecasts over 2.5 days, overlapping with the accident period. Since the emphasis is on the variance of the dispersion due to the variance in the forecasts, we restrict ourself to the free atmosphere, as mentioned above. The effect of differences between the meteorological forecasts is investigated for the different particle sizes. Large deviations from the results obtained with the deterministic forecast are found. In Sects. 2 and 3 the basic equation of motion of particles, and an overview of the database and numerical methods will be presented, respectively. Section 4 gives an overview of the 3-D position of the particles in each ensemble forecast. In Sects. 5 and 6 the vertical and horizontal distribution of the particles are presented, and Sect. 7 provides some statistical measures to quantify the separation of dispersion calculations in the ensemble forecasts. A summary and discussion is given in Sect. 8 .

\section{Equations of motion}

The motion of a small but heavy spherical particle is determined by the sum of the gravity and the Stokes drag. Buoyancy is negligible since it is proportional to the ratio of the density $\rho$ of air and $\rho_{\mathrm{p}}$ of the particle (which is less than $1 / 1000)$. The dimensionless form of the Newton equation is

St $\ddot{\boldsymbol{r}}_{\mathrm{p}}(t)=\dot{\boldsymbol{r}}_{\mathrm{p}}(t)-\boldsymbol{v}(\boldsymbol{r}, t)-w_{\text {term }} \boldsymbol{n}$,

where $\dot{\boldsymbol{r}}_{\mathrm{p}}$ is the velocity of the particle and $\boldsymbol{v}(\boldsymbol{r}, t)$ is the velocity of the ambient air at the location $r$ of the particle at time $t$, while $w_{\text {term }}$ is the terminal velocity in still fluid, and $\boldsymbol{n}$ is a unit vector pointing upwards. The particle acceleration $\ddot{\boldsymbol{r}}_{\mathrm{p}}(t)$ is multiplied by the Stokes number (Maxey and Riley, 1983):

$S t=\frac{2 r^{2} U}{9 v L} \frac{\rho_{\mathrm{p}}}{\rho}$,

where $L$ and $U$ represent a distance and velocity, respectively, characteristic to the flow, and $v$ is the kinematic viscosity of air. Since we are interested in phenomena on length scales $L \sim 10-1000 \mathrm{~km}$, and with wind speeds $U \sim$ $1-50 \mathrm{~m} \mathrm{~s}^{-1}$, the Stokes number for $v \approx 10^{-5} \mathrm{~m}^{2} \mathrm{~s}^{-1}$ and $\rho_{\mathrm{p}}=2000 \mathrm{~kg} \mathrm{~m}^{-3}$ is $S t \leq 2 \times 10^{-5}$. The left-hand side of Eq. (1) can thus be neglected, and the motion takes place practically under the balance of the Stokes drag and gravity. In other words, the particle velocity becomes immediately equal to the terminal velocity superimposed on the wind velocity. The equation of motion can then be written in the following form:

$\dot{\boldsymbol{r}}_{\mathrm{p}}(t) \equiv \boldsymbol{v}_{\mathrm{p}}=\boldsymbol{v}(\boldsymbol{r}, t)-w_{\text {term }} \boldsymbol{n}$.

The Stokesian terminal velocity for particles of radius $r$ (with $r<10 \mu \mathrm{m}$ ) is

$w_{\text {term }}=\frac{2}{9} r^{2} \frac{\rho_{\mathrm{p}}}{\rho \nu} g$, 
where $g$ denotes the gravitational acceleration.

Since the meteorological fields used for the simulations (see Sect. 3) are given on pressure levels, we determine trajectories in pressure coordinates. The vertical component of Eq. (3) is

$\dot{z}_{\mathrm{p}}(t) \equiv w_{\mathrm{p}}=w\left(\boldsymbol{r}_{\mathrm{p}}(t), t\right)-w_{\text {term }}$.

Similar to Eq. (5), the vertical motion of a particle in pressure coordinates can be written as

$\frac{d p\left(\boldsymbol{r}_{\mathrm{p}}(t), t\right)}{\mathrm{d} t} \equiv \omega_{\mathrm{p}}=\omega\left(\boldsymbol{r}_{\mathrm{p}}(t), t\right)-\omega_{\mathrm{term}}$,

where $\omega_{\text {term }}$ (applying the hydrostatic approximation) is (Haszpra and Tél, 2011)

$\omega_{\text {term }}=-\frac{2}{9} r^{2} \frac{\rho_{\mathrm{p}}}{v} g^{2}$.

Since in the horizontal direction spherical coordinates are used, we solve the following equations to determine the position of the particles:

$$
\begin{aligned}
\frac{d \lambda_{\mathrm{p}}}{\mathrm{d} t} & =\frac{u\left(\lambda_{\mathrm{p}}, \varphi_{\mathrm{p}}, p_{\mathrm{p}}, t\right)}{R_{\mathrm{E}} \cos \varphi_{\mathrm{p}}}, \\
\frac{d \varphi_{\mathrm{p}}}{\mathrm{d} t} & =\frac{v\left(\lambda_{\mathrm{p}}, \varphi_{\mathrm{p}}, p_{\mathrm{p}}, t\right)}{R_{\mathrm{E}}}, \\
\frac{d p_{\mathrm{p}}}{\mathrm{d} t} & =\omega\left(\lambda_{\mathrm{p}}, \varphi_{\mathrm{p}}, p_{\mathrm{p}}, t\right)-\omega_{\text {term }},
\end{aligned}
$$

where $\lambda_{\mathrm{p}}$ and $\varphi_{\mathrm{p}}$ are the longitude and latitude coordinates of a particle, $p_{\mathrm{p}}(t) \equiv p\left(\boldsymbol{r}_{\mathrm{p}}(t), t\right)$ is the pressure coordinate of a particle along its path, and $R_{\mathrm{E}}$ is the radius of the Earth. The limit of $r=0$ can be considered as the passive advection dynamics for air parcels since $\omega_{\text {term }}=w_{\text {term }}=0$ and hence from Eq. (3) $\dot{\boldsymbol{r}}_{\mathrm{p}}(t)=\boldsymbol{v}(\boldsymbol{r}, t)$ in this case.

\section{Data and methods}

In order to study the role of the different meteorological forecasts in dispersion calculation, we consider a hypothetical emission initiated by distributing several particles uniformly in a horizontal square in the free atmosphere.

The wind field data $(u, v, \omega)$ in Eq. (8a)-(8c) are taken from the forecasts of the European Centre for MediumRange Weather Forecasts (ECMWF) (Molteni et al., 1996; Buizza et al., 1999; Leutbecher and Palmer, 2008). For the meteorological situation, see Sect. 4. In our simulations based on Eq. (8a)-(8c), the only source of uncertainty is in the variance among the wind fields of the ensemble members. We simulate the dispersion of aerosol particles using a deterministic forecast and a 50-member ensemble forecast for a 2.5-day period starting at 00:00 UTC on 12 March 2011. The 51st member, the control forecast with non-perturbed initial conditions, is also available. The meteorological variables are given at pressure levels $(1000,925,850,700,500$,
$400,300,250,200,100,50,10 \mathrm{hPa})$ on a $0.125^{\circ} \times 0.125^{\circ}$ and on a $0.25^{\circ} \times 0.25^{\circ}$ horizontal grid for the deterministic forecast and for the ensemble forecast (including the control forecast), respectively. The time resolution is $3 \mathrm{~h}$. We are interested in the free atmospheric dispersion of air pollutants. Therefore, the simulations are stopped below (from the direction of smaller pressures) the $850 \mathrm{hPa}$ pressure level (about $1.5 \mathrm{~km}$ on average). In the region monitored, the impact of turbulent diffusion and wet deposition is negligible; hence for the dispersion calculation, wind speed data are sufficient. In the ensemble forecasts, the vertical velocity of the air is not archived on the 400, 300 and $250 \mathrm{hPa}$ levels. Therefore, $\omega=0$ was applied as a first estimate on these levels. This approximation does not appear to be a strong restriction since according to the deterministic forecast hardly any particles reach this region.

In order to compute trajectories, the wind data on the regular grid are interpolated to the location of the particles (using bicubic spline interpolation in horizontal and linear interpolation in vertical, and in time). The equations of motion Eq. (8a)-(8c) are solved by Euler's method with a time step of $\Delta t=5.625 \mathrm{~min}$. We chose this time step because a further reducing of it does not lead to further notable changes in the dispersion results. In view of this observation, we conclude that the variability of the results is physically reliable.

\section{General overview of the results}

Aerosol particles of various sizes of density $\rho_{\mathrm{p}}=$ $2000 \mathrm{~kg} \mathrm{~m}^{-3}$ and up to radius $r=10 \mu \mathrm{m}$ are considered. A 2D set of $n_{0}=300^{2}$ aerosol particles of a given size is released on the $500 \mathrm{hPa}$ level on 12 March 2011 at 00:00 UTC above Fukushima (the center of the cloud is $\lambda=141^{\circ}, \varphi=37.5^{\circ}$ ). The initial distribution is uniform over a square of size of $1^{\circ} \times 1^{\circ}(\sim 100 \times 100 \mathrm{~km})$. Note that with this choice of a 2$\mathrm{D}$ initial distribution, we do not test variances in the initial vertical wind field. The use of a columnar initial distribution can lead to only stronger particle dispersion variabilities due to the vertical wind shear. We shall see that dispersion variabilities are strong enough even with this restriction in the initial distribution. The particle cloud is followed for 2.5 days. Figure 1 presents the 3-D and top view distribution of all the 90000 particles of radius of $r=1,4$ and $10 \mu \mathrm{m}$ over the Pacific Ocean after 2.5 days. The aerosol particle distribution is filamentary in each ensemble member (marked by different colors) since advection dynamics of particles is typically chaotic in the atmosphere (Haszpra and Tél, 2011). The widely stretched shape of the pollutant clouds can be interpreted in terms of the meteorological fields of the time period investigated (Fig. 2). A characteristic feature is a jet located east to Japan in the Pacific region during these days (Fig. 2a, c and e). First, the particles travel to the east with the strong westerly flows of the jet (Fig. 2a) and are advected downwards in the meantime since $\omega>0$ east to Japan (see 

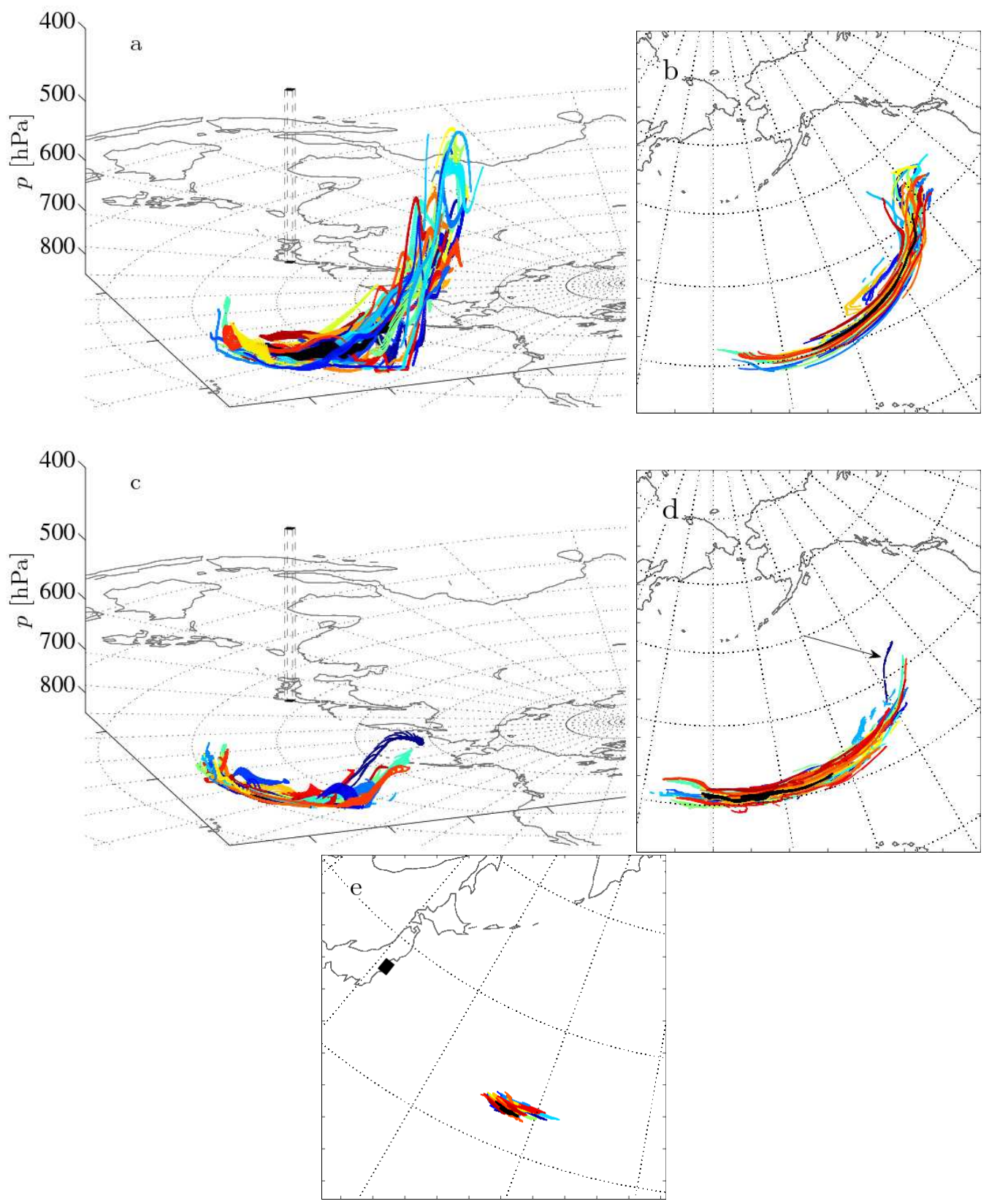

Fig. 1. The distribution of particles of $r=1 \mu \mathrm{m}(\mathbf{a}, \mathbf{b})$ and of $r=4 \mu \mathrm{m}(\mathbf{c}, \mathbf{d})$ in 3-D (a, c) and in a planar projection (b, d), respectively, after 2.5 days. Panel (e) shows the $r=10 \mu \mathrm{m}$ particles deposited on the $850 \mathrm{hPa}$ pressure level. Colors (blue to red) indicate the ensemble members; black is the deterministic forecast (overlaid on top of the others in panels $\mathbf{b}, \mathbf{d}, \mathbf{e}$ ). Black rectangles mark the initial condition above Fukushima and its surface-projected location. For the arrow in panel d, see text. For the ensemble members only every 20 th particle is plotted.

Fig. 2b). Above the Pacific Ocean, a cyclone passes towards the Californian coastline during the days of the simulation (Fig.2d and f). A considerable amount of the particles appears to be captured by the upwelling zone of this cyclone. Therefore, the altitude of the particles spreads considerably, mainly for the $r=1 \mu \mathrm{m}$ particles (Fig. 1a), around this geographical location. Owing to their much larger terminal velocity, all of the $r=10 \mu \mathrm{m}$ particles "deposit" on the bottom of the simulation (below the $850 \mathrm{hPa}$ level) within the investigated time period, and the deposition pattern has a much smaller extent than the area covered by the $r=1$ and $4 \mu \mathrm{m}$ particles (Fig. 1e).

Note that a wide zonal and vertical range of spread is found for the 50 forecasts (colors blue to red), especially for the 

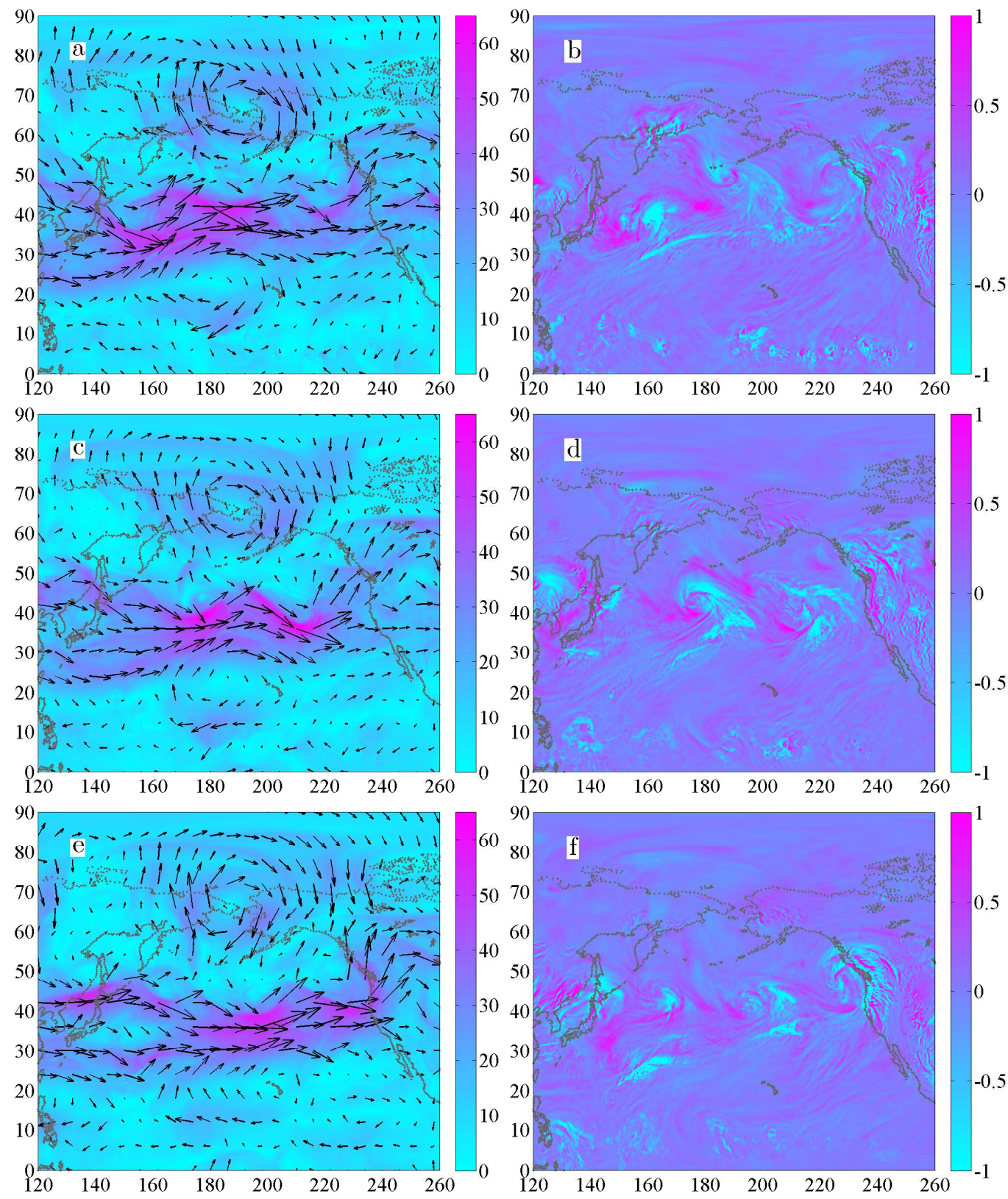

Fig. 2. The horizontal components $u, v\left[\mathrm{~m} \mathrm{~s}^{-1}\right](\mathbf{a}, \mathbf{c}, \mathbf{e})$ and the vertical component $\omega\left[\mathrm{Pa} \mathrm{s}^{-1}\right](\mathbf{b}, \mathbf{d}, \mathbf{f})$ of the wind speed at $500 \mathrm{hPa}$ on 12 March (a, b), 13 March (c, d), and 14 March (e, f) at 00:00 UTC in the deterministic forecast. Axis labeling corresponds to geographical coordinates in degree.

$r=1 \mu \mathrm{m}$ particles, while the dispersion prediction from the deterministic forecasts (black) is located inside the region covered by the ensembles. It is interesting that one of the ensemble members for $r=4 \mu \mathrm{m}$ (dark blue, marked by arrow in Fig. 1d) results in a much smaller cloud than the others, and it is also separated from them.

\section{Vertical distribution}

To gain an easily visualizable representation of the final vertical distribution, we split the free atmosphere into nine pressure layers of "height" of $50 \mathrm{hPa}$ and plot the particle proportion in each layer, irrespective of their horizontal coordinates. In Fig. 3 the distribution of particles of radius $r=1$ and $4 \mu \mathrm{m}$ is shown both in the ensemble members (colors blue to red) and in the deterministic forecast (black). In the lowest, 10th layer (below the black dashed lines representing the bottom 

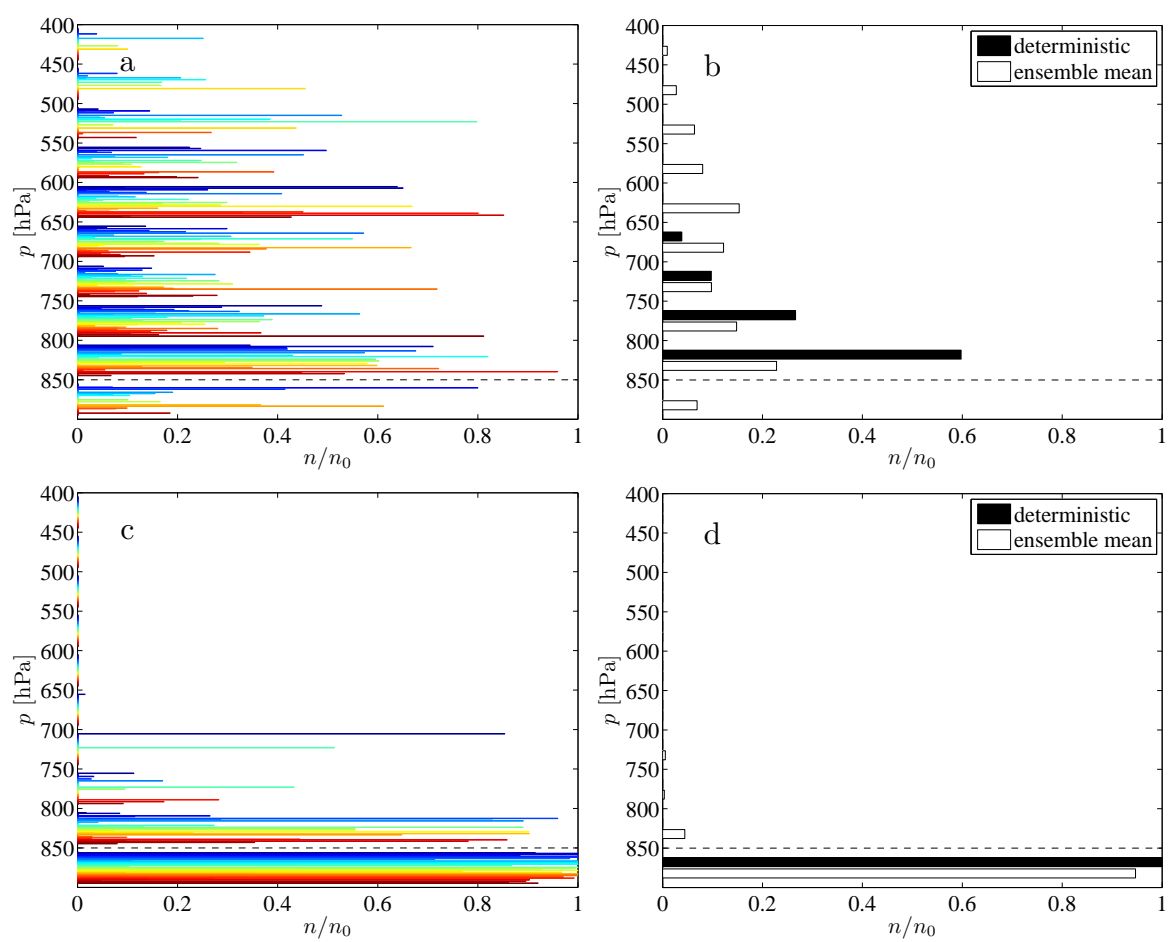

Fig. 3. The vertical distribution of the particles of $r=1 \mu \mathrm{m}(\mathbf{a}, \mathbf{b})$ and $r=4 \mu \mathrm{m}(\mathbf{c}, \mathbf{d})$ after 2.5 days coarse-grained in 10 layers. Colors in (a) and (c) indicate the 50 ensemble members; black and white in (b) and (d) mark the deterministic forecast and the ensemble mean, respectively. The black dashed lines illustrate the bottom level of the simulation range. $n / n_{0}$ denotes the proportion of particles in a given layer within a single forecast $\left(n_{0}=90000\right)$.

of the simulation region), we collected all the particles that stopped at $p_{\mathrm{p}} \geq 850 \mathrm{hPa}$. These are the particles deposited throughout the 2.5 days. So the bars below the dashed lines represent a cumulative particle proportion, in contrast to the free atmospheric bars that represent the position of the particles at the end of the observation period (after 2.5 days). The ensemble members have large variability, particularly for small-sized particles. For example for $r=1 \mu \mathrm{m}$ (Fig. 3a), the top layers $(400-450 \mathrm{hPa}$ and $450-500 \mathrm{hPa})$ include particles only in 5 and 8 ensemble members, respectively. For the middle and lower part of the free atmosphere (500-850 hPa), almost every ensemble member predicts some particles, but their proportions vary significantly from some $0.1 \%$ to about $90 \%$. For $r=4 \mu \mathrm{m}$ particles (Fig. 3c), in most ensemble members a large proportion reaches the bottom of the simulation region. A considerable part of the particles is predicted to end up in the lower layers. The "outlier" prediction (dark blue) mentioned in Sect. 4 has almost all the particles between the 700 and $750 \mathrm{hPa}$ levels, implying that this ensemble member represents a strongly atypical behavior.

Figure $3 b$ and $d$ illustrate the vertical distribution of the particles in the deterministic forecast compared to the ensemble mean in each layer. As Fig. 1 suggests, the ensemble mean exhibits a shallower but elongated histogram over the pressure layers. Particles from the deterministic forecast extend to a narrower vertical region. The deterministic forecast, for example, has no particles deposited on the bottom level for $r=1 \mu \mathrm{m}$.

In Fig. 4 the difference between the ensemble mean and the deterministic particle number is presented for 11 particle sizes $r=0-10 \mu \mathrm{m}$ in the $50 \mathrm{hPa}$ layers of the free atmosphere. In contrast to particles of finite radius, $r=0 \mu \mathrm{m}$ represents a gaseous contaminant with the same density as air as mentioned earlier. For small-sized particles, which remain in the atmosphere for several days, there are strong positive differences in the upper part of the simulation range (blue columns, $400-650 \mathrm{hPa}$ ), and negative deviations in the middle part. Below the bottom layer - where the deterministic forecast has no particles (Fig. 3b) - the ensemble mean is greater again. For large particles that mostly "deposit" within 2.5 days, the difference is much smaller. In the upper part of the atmosphere, the difference is 0 since neither the ensemble members nor the deterministic forecast predicts particles to be there.

\section{Horizontal distribution}

Besides the vertical variability, it is worth investigating the horizontal distribution of the particles. The particle number in each air column of a given size is determined in the 


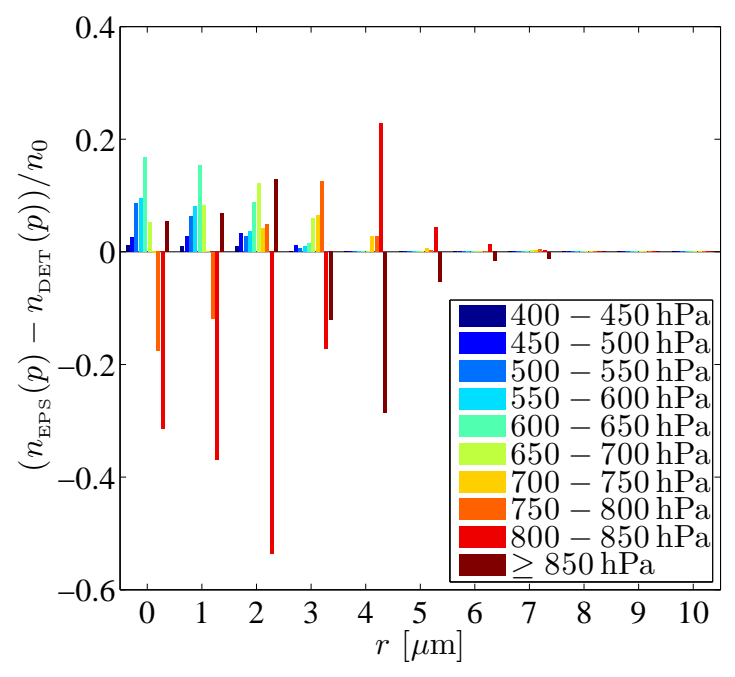

Fig. 4. The difference in the proportion of particles in the layers (colors) between the ensemble mean and the deterministic forecast for $r=0-10 \mu \mathrm{m}$.

deterministic forecast, as well as in the ensemble forecast, irrespective of the height. In risk assessment it is rather useful to estimate the extent of the area where the concentration of air pollutants exceeds a certain threshold. In this spirit, in Fig. 5a a black zone illustrates the region where in the deterministic forecast the concentration of the particles is larger than $c_{\text {th }}=1 / n_{0}=0.0011 \%$ of the total particle number. Colored contours demonstrate areas where a given proportion of the ensemble members predicts concentrations above the same threshold. Analogously, Fig. 5b exhibits the same concentrations in the "deposition field" for particles that reach the $850 \mathrm{hPa}$ level within 2.5 days. Hardly any of these heavy particles remain in the free atmosphere, and hardly any of the light ones become deposited. Therefore, no deposition field is shown for $r=1 \mu \mathrm{m}$, and no free atmosphere concentration for $r=10 \mu \mathrm{m}$. The area where, according to at least one ensemble member $(2 \%)$, the threshold is exceeded expands to a 5-10 times larger region than the area from the deterministic forecast, both for air column concentration (a) and in the "deposition" field (b).

The concentration of the particles from the deterministic forecast (gray scale) and the ensemble mean concentration (colored contours) are given in Fig. 6 in the air (a) and in the bottom layer (b). The locations of maxima are not exactly the same in the two cases as can very clearly be seen in Fig. 6b. The averaging procedure leads to lower maxima for small particles $(0.0078$ in the ensemble mean, while the global maximum is 0.0287 in the deterministic forecast). For $r=10 \mu \mathrm{m}$, there is a smaller difference in the extreme values (0.0772 and 0.1363 , respectively) (Fig. 6b). The reason for this is the weak separation of these particles due to the shorter time they spent in the free atmosphere before reaching the bottom level.

\section{Statistical measures}

\subsection{Root-mean-square distance among the particle clones}

In order to quantify the role of the various meteorological forecasts for the same time period, we calculate the rootmean-square horizontal and vertical distance of each particle from its own clones, i.e., particles starting from the same initial condition, in the $N=50$ ensemble members, in the air $\left(p_{\mathrm{p}, i}<p_{0}=850 \mathrm{hPa}\right)$ and also in the deposition field $\left(p_{\mathrm{p}, i} \geq p_{0}\right)$. For one particle we define the root-mean-square distance of each particle from its own clones, the clone distance for short:

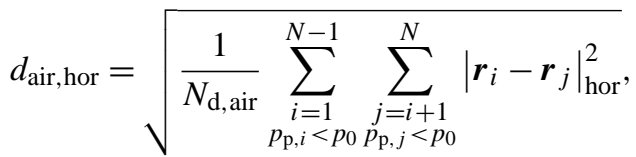

$$
\begin{aligned}
& d_{\text {air, vert }}=\sqrt{\frac{1}{N_{\mathrm{d}, \text { air }}} \sum_{\substack{i=1 \\
p_{\mathrm{p}, i}<p_{0}}}^{N-1} \sum_{\substack{j=i+1 \\
p_{\mathrm{p}, j}<p_{0}}}^{N}\left|\boldsymbol{r}_{i}-\boldsymbol{r}_{j}\right|_{\text {vert }}^{2}}, \\
& d_{\text {dep, hor }}=\sqrt{\frac{1}{N_{\mathrm{d}, \mathrm{dep}}} \sum_{\substack{i=1 \\
p_{\mathrm{p}, i} \geq p_{0}}}^{N-1} \sum_{\substack{j=i+1 \\
p_{\mathrm{p}, j} \geq p_{0}}}^{N}\left|\boldsymbol{r}_{i}-\boldsymbol{r}_{j}\right|_{\text {hor }}^{2}} \text {, }
\end{aligned}
$$

where the positions of clone $i$ and $j$ are marked by $\boldsymbol{r}_{i}$ and $\boldsymbol{r}_{j}$, and $N_{\mathrm{d} \text {, air }}$ and $N_{\mathrm{d} \text {,dep }}$ denote the number of clone pairs in the atmosphere and in the deposition field, respectively $\left(N_{\mathrm{d} \text {,air }}\right.$ and $N_{\mathrm{d}, \mathrm{dep}}$ are less than or equal to $\left.(N-1) N / 2\right)$. It is worth emphasizing that these quantities are undefined, or formally 0 in the deterministic forecast, their values can thus be considered as a measure of the variance of the particle distribution in the ensemble forecast.

In the horizontal direction, we take the spherical distance of the clone pairs:

$\left|\boldsymbol{r}_{i}-\boldsymbol{r}_{j}\right|_{\text {hor }}=$

$\arccos \left(\sin \varphi_{i} \sin \varphi_{j}+\cos \varphi_{i} \cos \varphi_{j} \cos \left(\lambda_{i}-\lambda_{j}\right)\right) \frac{180}{\pi} 111.1[\mathrm{~km}]$,

and the vertical distance is computed as

$\left|\boldsymbol{r}_{i}-\boldsymbol{r}_{j}\right|_{\mathrm{vert}}=\left|p_{\mathrm{p}, i}-p_{\mathrm{p}, j}\right|[\mathrm{Pa}]$.

Then the distributions of $d_{\mathrm{air}, \mathrm{hor}}, d_{\mathrm{air}, \mathrm{vert}}$, and $d_{\mathrm{dep}, \text { hor }}$ are considered for the $n_{0}=90000$ particles monitored (Fig. 7). We determine the statistics of the root-mean-square distance for clones over all particles, distinguishing those that remain in the atmosphere (Eq. 9a and 9b) and that deposit (Eq. 9c). Therefore, the larger the particle size, the less the number of clones in the air (the more the number of clones in the deposition field). Table 1 presents the minimum and maximum number of particle clones that remain in the atmosphere for 2.5 days, respectively, and the proportion of particle groups of clones $n / n_{0}$ in which at least 2 out of the 50 clones remain 
Table 1. Minimum and maximum number of particle clones $\left(n_{\text {clone, min }}, n_{\text {clone, max }}\right)$ among the $n_{0}$ particles remaining in the atmosphere for particle radius $r . n$ denotes the number of particles having at least two clones remaining in the atmosphere over 2.5 days.

\begin{tabular}{lrrrrrrrrrrr}
\hline$r[\mu \mathrm{m}]$ & 0 & 1 & 2 & 3 & 4 & 5 & 6 & 7 & 8 & 9 & 10 \\
\hline$n_{\text {clone, } \min }$ & 40 & 40 & 36 & 20 & 8 & 2 & 2 & 2 & 0 & 0 & 0 \\
$n_{\text {clone, } \max }$ & 50 & 50 & 49 & 46 & 26 & 21 & 21 & 21 & 0 & 0 & 0 \\
$n / n_{0}$ & 1.000 & 1.000 & 1.000 & 1.000 & 1.000 & 0.668 & 0.147 & 0.027 & 0.000 & 0.000 & 0.000 \\
\hline
\end{tabular}
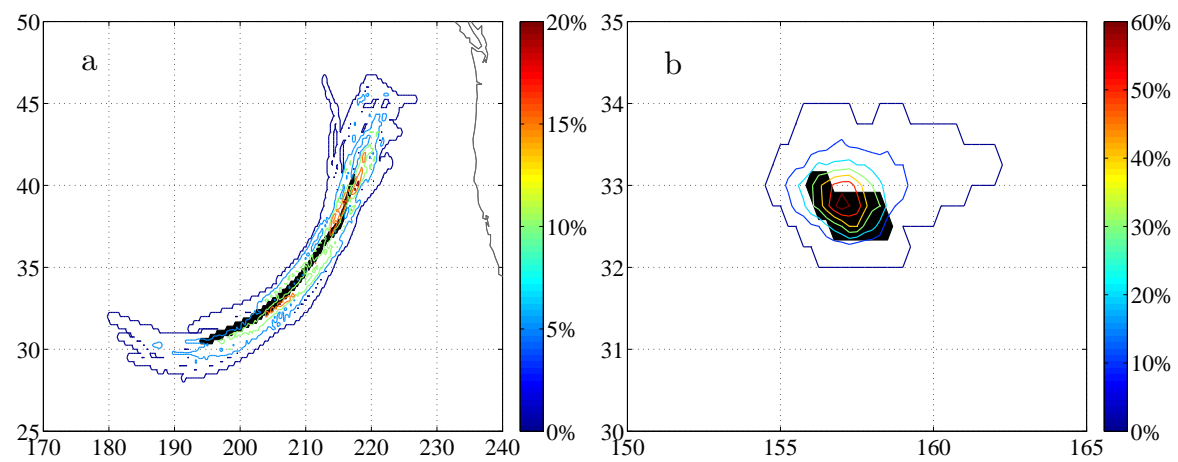

Fig. 5. Colored contours (at 2, 5, 10, 15, $20 \%$ in a, and at $2 \%, 10 \%, 20 \%, \ldots, 60 \%$ in b) indicate the percentage of the ensemble dispersion simulations predicting a "concentration" above the threshold $c_{\text {th }}=0.0011 \%$ after 2.5 days in a $0.25^{\circ} \times 0.25^{\circ}$ (a) air column for $r=1 \mu \mathrm{m}$ and (b) cell for $r=10 \mu \mathrm{m}$ "deposited" particles. Black cells refer to the results based on the deterministic forecast where the concentration is above $c_{\text {th }}$. Axis labeling corresponds to geographical coordinates in degree.

in the atmosphere (so the root-mean-square distance between the clones can be determined). Table 2 is the same for the deposited particles. For $r=8,9$ and $10 \mu \mathrm{m}$, none of the ensemble members has at least two particles in the free atmosphere.

For small particles the median of the clone distance in the horizontal direction in air and on the bottom level is about 1000 and $1500 \mathrm{~km}$, respectively. This appears to be a remarkably large distance compared to the average displacement from Fukushima, which is about $5000 \mathrm{~km}$. For large $r$, it reduces to some hundreds of kilometers (Fig. 7a). The horizontal spread of the same particle due to meteorological variability (represented by the ensemble members) is thus smaller for large particles, but even these smaller values are the size of a smaller country.

As a consequence of the height of the troposphere, the clones cannot separate so much in the vertical. The median of $d_{\text {air,vert }}$ ranges between 50 and $150 \mathrm{hPa}$ (about $0.5-2 \mathrm{~km}$ ) for $r=0-5 \mu \mathrm{m}$, and it decreases to some hundreds of meters or less for $r=6 \mu \mathrm{m}$. It is worth emphasizing that for $r=5-7 \mu \mathrm{m}$ we have only $2-21$ clones to estimate the rootmean-square distance among them, and the proportion of the available particle groups in the total of 90000 groups drops to 0.147 and 0.027 (Table 1).

\subsection{Center of mass and standard deviation}

The center of mass $\left(\lambda_{\mathrm{CM}}, \varphi_{\mathrm{CM}}, p_{\mathrm{CM}}\right)$ of each particle cloud (of $n_{0}$ particles) of the ensemble members and in the deterministic forecast are calculated. The center of mass of each ensemble member in the horizontal is calculated in a 3D Cartesian coordinate system (the $(0,0,0)$ of which is the center of the Earth). Then the coordinates are projected to the surface of Earth in the latitude-longitude coordinate system. ${ }^{1}$ In addition, the standard deviations $(\sigma)$ are also calculated both in horizontal and vertical using spherical distances and pressure coordinate differences, respectively, between the centers of mass and the particles in the different ensemble members. Furthermore, these quantities over the whole ensemble forecast are determined, too, both among the particles remaining in the atmosphere for 2.5 days and among the particles that have landed on the bottom level of $850 \mathrm{hPa}$.

The centers of mass of the ensemble members extend to a large area, and the spread of the centers of mass decreases with particle radius. Fig. 8a illustrates the horizontal location of the center of mass of each ensemble member for $r=1 \mu \mathrm{m}$ both for particles in the air (blue) and in the deposition field (red). Note that not every ensemble member has deposited particles (see Fig. 3a), so there are less red circles than blue (e.g., all the particles of the control forecast and the deterministic forecast remained in the air). The standard

\footnotetext{
${ }^{1}$ In this meteorological situation, because the particle clouds extend only slightly in the meridional direction, the projected coordinates differ at most $1 \%$ from that when the horizontal component of the center of mass is determined by averaging the longitudinal and latitudinal coordinates of the particles.
} 


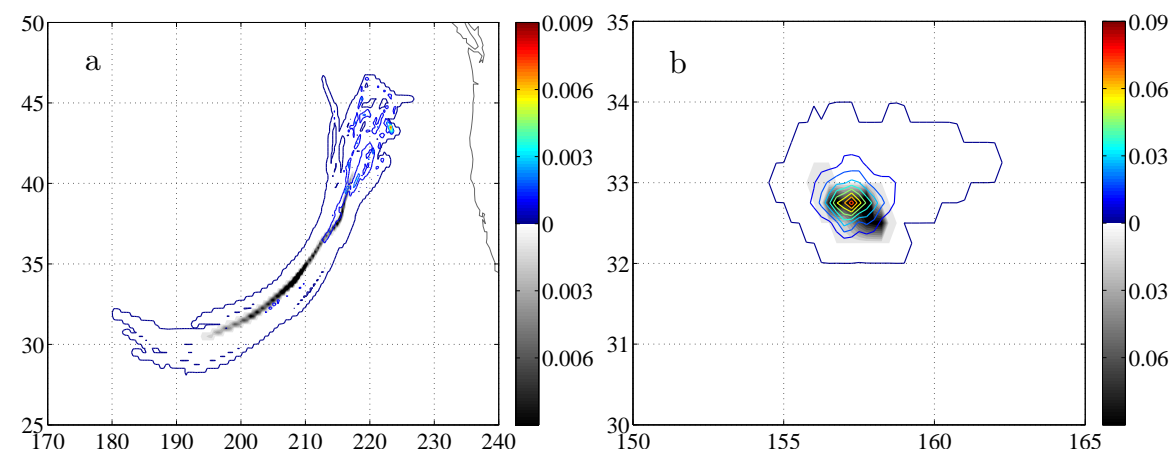

Fig. 6. Colored contours and gray contours indicate the ensemble mean and deterministic "concentration", respectively, in a $0.25^{\circ} \times 0.25^{\circ}$ (a) air column for $r=1 \mu \mathrm{m}$ and (b) cell for $r=10 \mu \mathrm{m}$ "deposited" particles after 2.5 days. Axis labeling corresponds to geographical coordinates in degree.
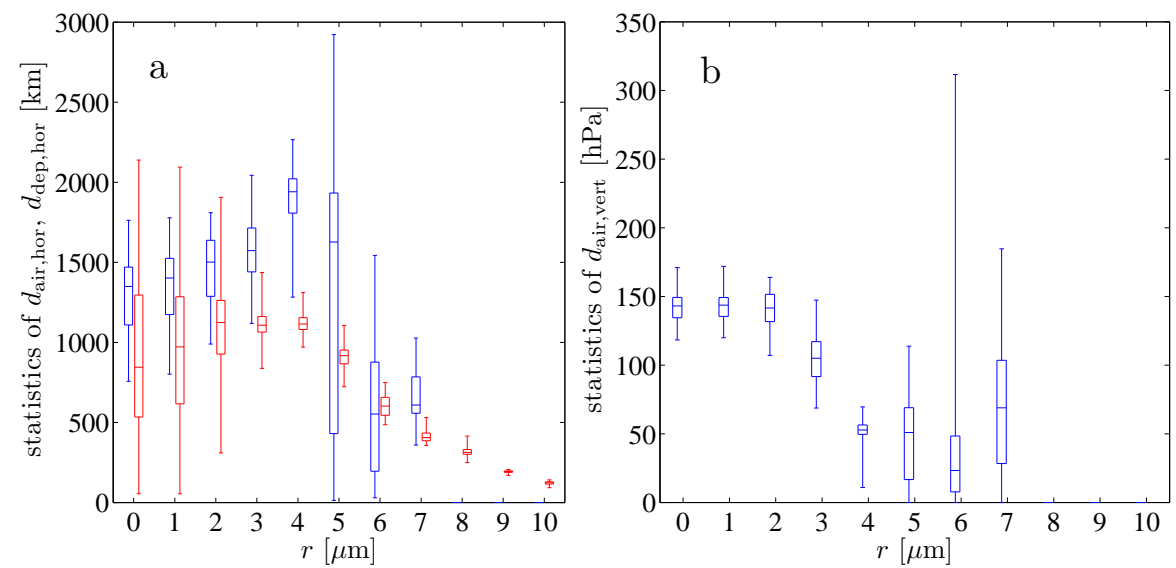

Fig. 7. The statistics of the root-mean-square clone distances $d_{\text {air,hor }}$ and $d_{\text {dep, hor }}$ in horizontal (a) and $d_{\text {air,vert }}$ in vertical (b) of the particles in the 50 ensemble members after 2.5 days averaged over the particles that remain in the atmosphere (blue) or deposit (red). Minima and maxima (horizontal line segments), lower and upper quartiles (the bottom and the top of the boxes), and medians (horizontal lines in the boxes) are plotted.

deviations of the particles in the clouds vary in a wide range (e.g., from $35 \mathrm{~km}$ to $960 \mathrm{~km}$ for $r=1 \mu \mathrm{m}$ particles in the air, and from $10 \mathrm{~km}$ to $950 \mathrm{~km}$ in the deposition field). In Fig. 8a the radii of the circles are proportional to the standard deviation around the center of mass coordinates within an ensemble member. In order to avoid a strong overlap of these circles, the radii are chosen to be 70 times smaller than the real values.

It is interesting to compare the results obtained in the fineresolution deterministic forecast (DET) and in the coarseresolution control forecast $(\mathrm{CF})$. The distance between the center of mass of the DET and CF cloud is about $750 \mathrm{~km}$. The difference can be interpreted as the error due to using a spatial grid twice the size of that of the deterministic forecast. The distance between the centers of mass of the furthest clouds (i.e., the impact of using the perturbed forecasts) is about 4.5 times greater $(3375 \mathrm{~km})$.
As expected on the basis of Fig. 3, the dispersion calculation using the deterministic forecast predicts for small particles the center of mass of the pollutant cloud lying lower than the ensemble mean center of mass (see Fig. 8b). The mean vertical component of the center of mass of the ensemble members is not necessarily the same as if we considered the mean of all the particles of the whole 50-member ensemble forecast, because the ensemble members may include various number of particles in the air.

The statistics over the ensemble members of the standard deviation around the center of mass for particles of different sizes can be seen in Fig. 9. For $r=0-4 \mu \mathrm{m}$ particles in the horizontal direction, it reaches some hundred kilometers, while for larger particles it is an order of magnitude smaller. The vertical spread of the particles varies between 10 and $80 \mathrm{hPa}(\sim 0.1-1.2 \mathrm{~km})$ and less than $10 \mathrm{hPa}$. Note that the trends in Figs. 9 and 7 are similar: the root-mean-square distance of the particle clones (from different forecast members) 
Table 2. Minimum and maximum number of particle clones $\left(n_{\text {clone, min }}, n_{\text {clone, max }}\right)$ among the $n_{0}$ particles depositing within 2.5 days for particle radius $r . n$ denotes the number of particles having at least two clones being deposited over 2.5 days.

\begin{tabular}{lrrrrrrrrrrr}
\hline$r[\mu \mathrm{m}]$ & 0 & 1 & 2 & 3 & 4 & 5 & 6 & 7 & 8 & 9 & 10 \\
\hline$n_{\text {clone, } \min }$ & 0 & 0 & 0 & 4 & 24 & 43 & 46 & 47 & 49 & 50 & 50 \\
$n_{\text {clone, } \max }$ & 10 & 10 & 14 & 30 & 42 & 50 & 50 & 50 & 50 & 50 & 50 \\
$n / n_{0}$ & 0.775 & 0.860 & 0.998 & 1.000 & 1.000 & 1.000 & 1.000 & 1.000 & 1.000 & 1.000 & 1.000 \\
\hline
\end{tabular}
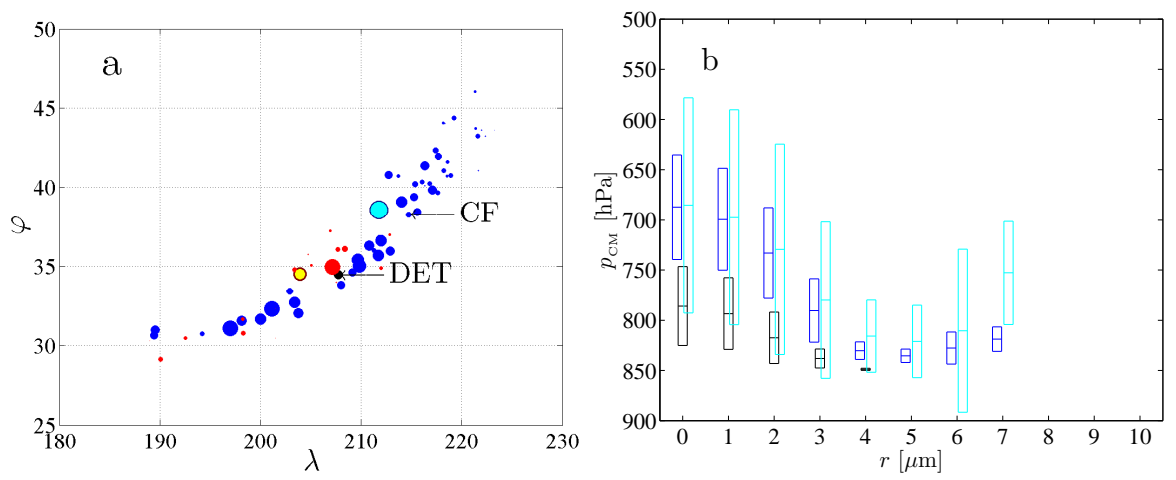

Fig. 8. (a) The horizontal location of the center of mass of the pollutant clouds in the atmosphere (blue) and in the deposition field (red) for the 50 ensemble members after 2.5 days. The radii of the circles are proportional to the standard deviations. DET and CF denote the deterministic forecast (black) and the control forecast, respectively, and the cyan (yellow) dot with dark edge marks the center of mass and standard deviation of all particles of the 50 ensemble members that remain in the atmosphere (deposit). (b) The mean vertical coordinate of the center of mass of the ensemble members (lines in the boxes) with the mean standard deviation (the bottom and the top of the boxes) (blue), and those for the whole ensemble forecast (cyan) and for the deterministic forecast (black).

is in positive correlation with the spread of the individual clouds. Considering the total number of the particles of the whole ensemble forecast $\left(50 \times n_{0}\right)$, the standard deviation of the particles that are located in the atmosphere after 2.5 days exceeds $1000 \mathrm{~km}$ in the horizontal and reaches $80-100 \mathrm{hPa}$ $(\sim 1.2-1.5 \mathrm{~km})$ in the vertical (filled circles in Fig. 9).

\section{Summary and discussion}

In risk assessment it is rather useful to estimate the extent of the area where the concentration of air pollutants exceeds a certain threshold. We have demonstrated here that in an ensemble forecast this area might largely exceed that obtained from a deterministic forecast: the smaller the particles, the stronger the effect.

Both the deterministic forecast and the ensemble forecast provide concentration distributions with pronounced extrema, the locations of which differ significantly at the end of the observation period. In the ensemble mean, the distribution becomes smoother than that of the deterministic case, suggesting that instead of the extrema of the ensemble mean, a proper representation of a worst case scenario requires the consideration of the local maxima in the whole ensemble.

We have demonstrated that the root-mean-square distance of any particle from its own clones in the ensemble members can reach values on the order of thousand kilometers, and both the location of the center of mass of the clouds and its standard deviation can vary in a wide range. That indicates that an investigation of the dispersion of aerosol particles in the spirit of ensemble forecast contains useful hints for the improvement of risk assessment.

In order to compare the variability of the Lagrangian and Eulerian quantities in the 50 forecasts, the standard deviation of the magnitude of the horizontal wind speed $\sqrt{u^{2}+v^{2}}$ was determined at grid points. In the horizontal direction we choose the grid points where at least one ensemble member predicts any particles at any levels (grid points contained within the outermost contours in Fig. 5). For particles above $850 \mathrm{hPa}$, the pressure levels $850-400 \mathrm{hPa}$ are selected, and for deposited particles level $850 \mathrm{hPa}$ was used. $\sigma_{\mathrm{E}}^{2}$ denotes the average of $\left(u^{2}+v^{2}\right)-{\overline{\sqrt{u^{2}+v^{2}}}}^{2}$ over all ensemble members at a given grid point. $\overline{\sigma_{\mathrm{E}}}$ is the average of the Eulerian standard deviation over the grid points, and $D\left(\sigma_{\mathrm{E}}\right)$ is the standard deviation of this distribution.

For particles that remain in the air, $D\left(\sigma_{\mathrm{E}}\right) / \overline{\sigma_{\mathrm{E}}}=0.38$ for $r=1 \mu \mathrm{m}$. The variability in the Lagrangian extent of the clouds is somewhat larger: $D(\sigma) / \bar{\sigma}=0.56$. For the deposition field (the particles deposited throughout the 2.5 days), these values are $D\left(\sigma_{\mathrm{E}}\right) / \overline{\sigma_{\mathrm{E}}}=0.18$ and $D(\sigma) / \bar{\sigma}=0.50$ for $r=5 \mu \mathrm{m}$ particles. (This size is chosen since $r=5 \mu \mathrm{m}$ 

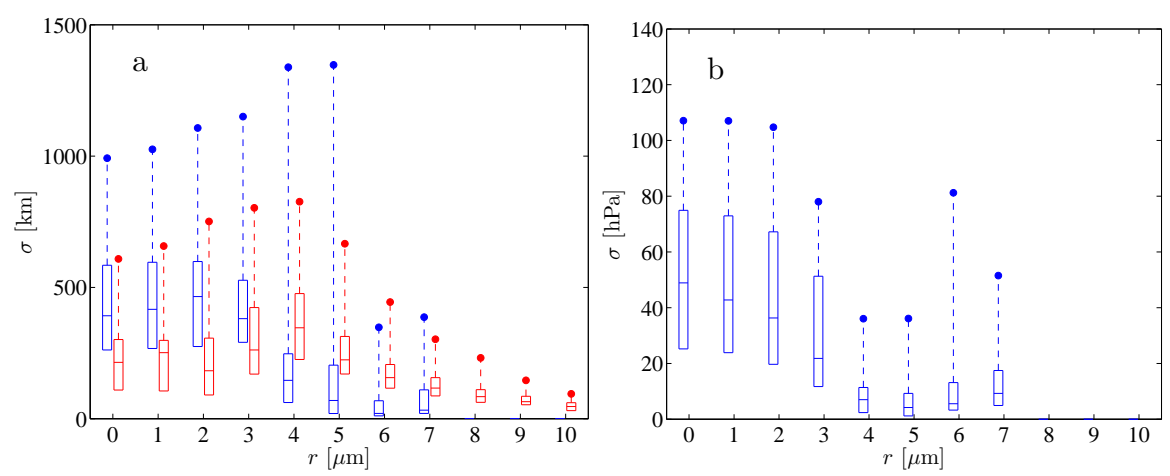

Fig. 9. The statistics of the standard deviation around the center of mass over the 50 ensemble members in horizontal (a) and in vertical (b) after 2.5 days averaged over the particles that remain in the atmosphere (blue) or deposit (red). Lower and upper quartiles (the bottom and the top of the boxes), and medians (horizontal lines in the boxes) are plotted. Filled circles denote the standard deviation over the whole ensemble forecast for particles that remain in the atmosphere or deposit.

particles are the ones whose majority reach the bottom level after 2.5 days. Note that in still air the same deposition time would belong to $r=7.5 \mu \mathrm{m}$, indicating the relevance of upand downwellings in the real atmosphere.) That means that the relative variance of the Eulerian and the Lagrangian ensemble statistics is typically on the same order of magnitude, but certain Lagrangian features might be 2-3 times larger.

Altogether our study suggests that an ensemble treatment of the pollutant dispersion of aerosol particles provides a more detailed exploration of extremes than the usual one based on a single weather forecast.

Acknowledgements. We are indebted to I. Ihász for providing us with the ECMWF data, and for valuable contributions. A. Horányi, I. Szunyogh and V. Homonnai are acknowledged for useful discussions. This work was supported by OTKA grants NK100296, K81933, K81975, K104666 and EU COST Action MP0806 on "Particles in Turbulence" and the ICMAT Severo Ochoa project SEV-2011-0087.

Edited by: C. López

Reviewed by: two anonymous referees

\section{References}

Buizza, R., Milleer, M., and Palmer, T. N.: Stochastic representation of model uncertainties in the ECMWF ensemble prediction system, Q. J. R. Meteorol. Soc., 125, 2887-2908, 1999.

Challa, V., Indrcanti, J., Baham, J., Patrick, C., Rabarison, M., Young, J., Hughes, R., Swanier, S., Hardy, M., and Yerramilli, A.: Sensitivity of atmospheric dispersion simulations by HYSPLIT to the meteorological predictions from a meso-scale model, Environ. Fluid Mechanics, 8, 367-387, 2008.

Dabberdt, W. F. and Miller, E.: Uncertainty, ensembles and air quality dispersion modeling: applications and challenges, Atmos. Environ., 34, 4667-4673, 2000.
Dop, H. V., Addis, R., Fraser, G., Girardi, F., Graziani, G., Inoue, Y., Kelly, N., Klug, W., Kulmala, A., Nodop, K., and Pretel, J.: ETEX: A Europian Tracer Experiment; observations, dispersion modelling and emergency response, Atmos. Environ., 32, 40894094, doi:10.1016/S1352-2310(98)00248-9, 1998.

Galmarini, S., Bianconi, R., Bellasio, R., and Graziani, G.: Forecasting the consequences of accidental releases of radionuclides in the atmosphere from ensemble dispersion modelling, J. Environ. Radioactivity, 57, 203-219, doi:10.1016/S0265931X(01)00017-0, 2001.

Galmarini, S., Bianconi, R., Klug, W., Mikkelsen, T., Addis, R., Andronopoulos, S., Astrup, P., Baklanov, A., Bartniki, J., Bartzis, J., Bellasio, R., Bompay, F., Buckley, R., Bouzom, M., Champion, H., D'Amours, R., Davakis, E., Eleveld, H., Geertsema, G., Glaab, H., Kollax, M., Ilvonen, M., Manning, A., Pechinger, U., Persson, C., Polreich, E., Potemski, S., Prodanova, M., Saltbones, J., Slaper, H., Sofiev, M., Syrakov, D., Sørensen, J., der Auwera, L., Valkama, I., and Zelazny, R.: Ensemble dispersion forecasting - Part I: concept, approach and indicators, Atmos. Environ., 38, 4607-4617, 2004.

Hágel, E. and Horányi, A.: The ARPEGE/ALADIN limited area ensemble prediction system: the impact of global targeted singular vectors, Meteorol. Z., 16, 653-663, 2007.

Haszpra, T. and Tél, T.: Volcanic ash in the free atmosphere: A dynamical systems approach, J. Physics: Conference Series, 333, 012008, doi:10.1088/1742-6596/333/1/012008, 2011.

Holt, T., Pullen, J., and Bishop, C. H.: Urban and ocean ensembles for improved meteorological and dispersion modeling of the coastal zone, Tellus, 61, 232-249, 2009.

Ihász, I., Üveges, Z., Mile, M., and Németh, C.: Ensemble calibration of ECMWF's medium-range forecasts, Időjárás, 114, 275286, 2010.

Kalnay, E.: Atmospheric modeling, data assimilation and predictability, Cambridge University Press, Cambridge, 2003.

Lee, J. A., Peltier, L. J., Haupt, S. E., Wyngaard, J. C., Stauffner, D. R., and Deng, A.: Improving SCIPUFF dispersion forecasts with NWP ensembles, J. Appl. Meteorol. Climatol., 48, 23052319, 2009.

Leutbecher, M. and Palmer, T.: Ensemble forecasting, J. Comput. Phys., 227, 3515-3539, 2008. 
Maxey, M. R. and Riley, J. J.: Equation of motion for a small rigid sphere in a nonuniform flow, Phys. Fluids, 26, 883-889, doi:10.1063/1.864230, 1983.

Molteni, F., Buizza, R., Palmer, T. N., and Petroliagis, T.: The ECMWF Ensemble Prediction System: Methodology and validation, Q. J. R. Meteorol. Soc., 122, 73-119, 1996.

Nasstrom, J. S. and Pace, J. C.: Evaluation of the effect of meteorological data resolution on Lagrangian particle dispersion simulations using the ETEX experiment, Atmos. Environ., 32, 41874194, doi:10.1016/S1352-2310(98)00180-0, 1998.

Scheele, M. P. and Siegmund, P. C.: Estimating errors in trajectory forecasts using ensemble predictions, J. Appl. Meteorol., 40, 1223-1232, 2001.

Sørensen, J. H.: Sensitivity of the DERMA long-range Gaussian dispersion model to meteorological input and diffusion parameters, Atmos. Environ., 32, 4195-4206, doi:10.1016/S13522310(98)00178-2, 1998.
Stohl, A., Forster, C., Frank, A., Seibert, P., and Wotawa, G.: Technical note: The Lagrangian particle dispersion model FLEXPART version 6.2, Atmos. Chem. Phys., 5, 2461-2474, doi:10.5194/acp-5-2461-2005, 2005.

Straume, A. G.: A more extensive investigation of the use of ensemble forecasts for dispersion model evaluation, J. Appl. Meteorol., 37, 425-445, 2001.

Straume, A. G., Koffi, E. N., and Nodop, K.: Dispersion modeling using ensemble forecasts compared to ETEX measurements, J. Appl. Meteorol., 37, 1444-1456, 1998.

Warner, T. T., Sheu, R.-S., Bowers, J. F., Sykes, R. I., Dodd, G. C., and Henn, D. S.: Ensemble Simulations with Coupled Atmospheric Dynamic and Dispersion Models: Illustrating Uncertainties in Dosage Simulations, J. Appl. Meteorol., 41, 488-504, 2002 . 